\title{
Research on video enhancement concentration and intelligent monitoring technology in Substation
}

\author{
Yuancheng Zhu ${ }^{1}$, Zhipeng Lei ${ }^{1}$, Xizhong $\mathrm{Li}^{1}$, Wei Zheng ${ }^{1}$ and Deyu Song ${ }^{2 *}$ \\ ${ }^{1}$ State Grid Yingkou Electric Power Supply Co., Ltd., Yingkou, Liaoning Province, 115000, China \\ ${ }^{2}$ State Grid Shenyang Electric Power Supply Co., Ltd., Shenyang, Liaoning Province, 110004, China
}

\begin{abstract}
With the development of intelligent theory of computer vision and the popularization of surveillance cameras, intelligent video analysis and surveillance technology has been widely used. The traditional video monitoring system will produce a large amount of video information in the application process, which is not conducive to the storage and reference of information, and it can not carry out realtime warning, and has poor robustness to the bad environment. In view of these disadvantages, this paper proposes an improved video enhancement and concentration method, which has a good effect in ensuring the video concentration ratio and the target loss rate, and can improve the shortcomings brought by manual reference. Aiming at the problem of real-time early warning, an intelligent video analysis method is proposed to realize the functions of event alarm and detection analysis. It is of great significance to improve the video monitoring management level of the substation and realize the unattended operation of the substation.
\end{abstract}

\section{Introduction}

Intelligent video analysis and video monitoring have been gradually integrated into various industries, especially in some places to ensure safe and stable operation, such as: unattended substation in power system [1].At present, the video monitoring system of the substation is mainly digital video monitoring system, which will generate a large amount of video data and occupy a large amount of network and storage resources in the process of operation; in the event of an accident or invasion, it is unable to generate automatic alarm and intelligent monitoring for early warning events directly, and it is unable to timely mobilize the relevant video, and in the later stage, it is also necessary to manually find the required information from the video data, resulting in waste of human resources. In addition, the traditional video monitoring system in special weather conditions, lack of clarity leads to weak video analysis, easy to miss important information[2].

Through intelligent analysis of video content, video enhancement and concentration technology can monitor and extract targets in different scenes, mine effective information from massive video information, and greatly improve the practicability of video monitoring system[3]. Intelligent video analysis technology can intelligently judge the target's behavior and track the target's track, so that it can timely alarm and take the initiative to prevent in case of abnormal conditions, and improve the timeliness of handling emergencies[4].
In this paper, an improved video enhancement and concentration method is proposed, which can quickly obtain the required information from the massive video data generated in the substation video monitoring system, this paper also proposes an intelligent video analysis method for intelligent processing of monitoring video, which can realize the functions of early alarm, simple after event tracing and accountability, retrieval and intelligent analysis and monitoring.

\section{Realization of video enhancement and concentration technology in Substation}

Video enhancement and concentration is one of the important research directions in the field of computer vision. Video enhancement and concentration usually includes four processes: target detection, target tracking, target fusion and condensed video generation. Target detection is the process of modeling the target and background to separate the foreground and background of the target; target tracking includes single scene and multi scene tracking, whose essence is to track the foreground of the target to obtain the moving track of the target; target fusion is to get the best moving track arrangement according to the extracted moving track of the target through certain algorithm processing; Condensed video generation is the process of generating the final video according to the optimal track extracted, combined with the set video background image[5]. In this chapter, the target fusion and the generation of 
condensed video in video enhancement and concentration are studied deeply, and a video concentration algorithm based on multiple constraints is proposed. Finally, the optimal arrangement of motion trajectory is obtained by differential evolution algorithm, and then the background image of the fused video is generated into condensed video, the process is shown in Figure 1.

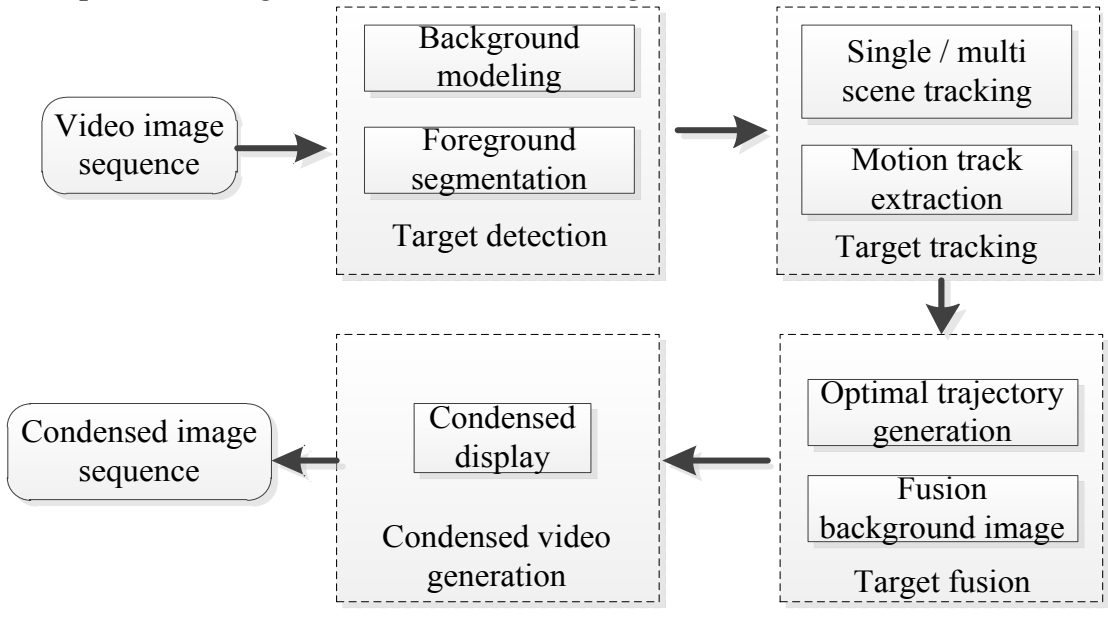

Figure 1. Enhance the video concentration process.

Firstly, a discrete motion model is established for the target's trajectory:

$$
T=\left\{\left(x_{1}^{1}, \cdots x_{1}^{d}, t_{1}\right),\left(x_{2}^{1}, \cdots x_{2}^{d}, t_{2}\right), \cdots,\left(x_{n}^{1}, \cdots x_{n}^{d}, t_{n}\right)\right\}
$$

Where: $T$ represents a track; $\left(x_{n}^{1}, \cdots x_{n}^{d}, t_{n}\right)$ represents a point in T-Time and d-dimensional space;

Based on the description method of trajectory motion model, the mapping relationship description is established for each motion trajectory extracted:

$$
f_{k}(x, y, t)=\left\{\begin{array}{l}
1,\left(x, y, t \in R_{t}^{k}\right) \\
0, \text { else }
\end{array}\right.
$$

In the condensed video clip, at time t, the set of target regions where the track of the $\mathrm{k}$-th target $l_{k}$ is $R_{t}^{k}$,after the track of each target is extracted, the set of tracks is obtained:

$$
\begin{gathered}
L=\left\{l_{1}, l_{2}, \cdots, l_{n}\right\} \\
T=\left\{t_{1}, \cdots, t_{k}\right\},\left(t_{k}=\left[l_{k}^{a}, l_{k}^{d}\right]\right)
\end{gathered}
$$

The trajectory set optimization problem can be transformed into the time transfer mapping problem for each trajectory. The energy function is introduced:

$$
E(M)=\alpha E_{a}+\beta E_{t}+(1-\alpha-\beta) E_{c}
$$

Where: $\alpha$ and $\beta$ are the set weight parameters, $E_{\alpha}$ is the length penalty function, $E_{t}$ is the consistency penalty function, $E_{c}$ is the pseudo collision penalty function. When $E(M)$ gets the minimum value, the optimal path combination can be found.

\section{Intelligent video monitoring technology}

Compared with mobile detection, intelligent video monitoring improves the anti-jamming ability and judgment accuracy. Intelligent video monitoring can determine the real active target in the video, while mobile detection can only distinguish whether the picture changes, but it can not distinguish whether the change comes from the target movement or background interference. Based on the enhancement of video concentration, this paper uses intelligent monitoring and analysis technology to embed different alarm rules in different scenes, when the active target is judged to violate the relevant rules, the monitoring system will start the corresponding alarm measures, including starting the local audible and visual alarm, remote monitoring center pop-up alarm video, alarm information, automatic saving, enhanced alarm video screen, etc. The overall technical roadmap is shown as follows: 


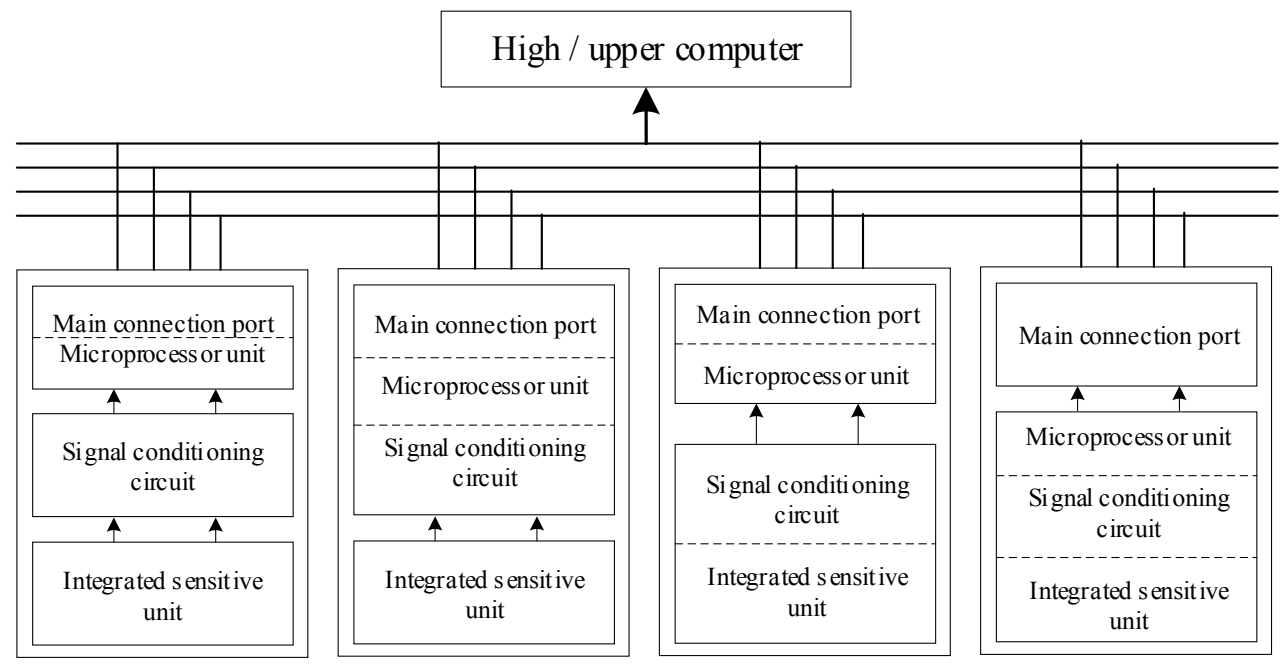

Figure 2. The Technical Roadmap

\subsection{Make alarm rules}

According to the special security requirements of unattended substation, the alarm rules and alarm levels are formulated, and the results of video image analysis are classified and uploaded, realize the functions of area intrusion detection, moving object crossing detection, personnel leaving area detection, moving direction detection, object left detection, object loss detection, personnel abnormal wandering detection, interference / damage / moving / blocking camera detection, etc. Different detection results are processed separately.

\subsection{Alarm control}

According to the established alarm rules, when the video identification is finished, corresponding alarm measures shall be started respectively, such as local audible and visual alarm, remote monitoring center pop-up alarm video, background monitoring host upload alarm information, etc. Then it can save the alarm video automatically and enhance the image, ensure the stable operation under the bad weather conditions such as rain, snow, wind and fog, and accurately identify various objects in the alarm area.

\section{Conclusion}

According to the current situation of unattended substation and special security requirements, this paper enhances the video concentration technology, and effectively combines it with intelligent detection technology, which is of great significance to improve the video monitoring management level of substation, improve the level of intrusion protection, and ensure the safe and stable operation of power grid.

\section{Acknowledgement}

Fund project: Science and technology project of State Grid: Research on video enhancement concentration and intelligent analysis and monitoring technology. (2019YF35)

\section{References}

1. Liang, G., Zhang, Y., Luo,B. (2018). Design of intelligent video information fusion monitoring system for substation. 2018 smart grid information construction seminarg.

2. Huang, K., Chen, X., Kang, Y., Tan, T. (2015). Overview of intelligent video monitoring technology. Journal of computer science, 20 (6), 1093-1118.

3. Wang, C. (2017). Overview of moving object detection methods in intelligent video monitoring system. Automation and instrumentation (3), 1-3.

4. Chen, C., Xu, L. Research on new intelligent video monitoring system for substation. East China Science and technology: Academic Edition (1), 194-194.

5. Shan, X. (2016). Surveillance video concentration based on target trajectory (Anhui University). 